\title{
The Efficiency of AMEn and TMEn Utilization for NE in Broiler Diets
}

\section{-Author(s)}

Yaghobfar $\mathrm{A}^{\prime}$

Animal Science Research Institute, I.R of Iran - Karaj.

\section{nail Address}

Corresponding author e-mail address Akbar Yaghobfar

Animal Science Research Institute, I.R of Iran - Karaj, Tehran - P. Box: 31585-1483.

Phone: (09826) 34430010

Email: yaghobfar@yahoo.com

\section{EKeywords}

AMEn, TMEn, NE, EER, PER.

\section{ABSTRACT}

The experiment was conducted to evaluate the feed intake, body weight gain, total energy $(\mathrm{kJ})$, protein $(\mathrm{g})$ intake, energy and protein efficiency ratio (EER, PER), net energy, and metabolic body weight, on two commercial broiler chickens (Arian and Ross 308 strains). Four treatmentsincluded diets formulated based onAMEn $(\mathrm{kJ} / \mathrm{g})$ Total Amino Acid (TAA) (T1), AMEn(kJ/g) Digestible Amino Acid (T2), TMEn $(\mathrm{kJ} / \mathrm{g})$ Total Amino Acid (T3), and TMEn ( $\mathrm{kJ} / \mathrm{g})$ Digestible Amino Acid (DAA) (T4) for commercial broilers chickens. The findings of the study indicated that AMEn or TMEn treatments yielded improved utilization of net energy in 42 days, but did not affect the efficiency of dietary AMEn and TMEn for net energy, diet energy and protein efficiency ratio (EER, PER) in broilers. Findings indicated that NE is a better predictor of poultry feeds than AMEn or TMEn. This is attributed to the fact that AMEn had a significant effect on NE and efficiency of AMEn or TMEn and $\mathrm{HI}$ (Heat Increment) for NE value. Finally, NE is the final objective of energy evaluation of feed and feedstuffs for poultry nutrition.

\section{INTRODUCTION}

It is important to estimateprecisely the energy value of feedstuffs and diets, either for least-cost formulation or for adapting feed supply to the metabolizable energy requirements of broilers. Unfortunately, such energy is not used with $100 \%$ of efficiency for production, because during metabolism, around $15 \%$ of the energy is wasted as heat, and this is commonly referred to as heat increment or specific dynamic action. In fact, net energy values vary with bird age, species, and production level; this poses a logistical problem during formulation. Between 0-21 days of age, it has been reported that about $84 \%$ of the metabolizable energy (ME) is available as net energy (NE), although bird-related factors and/or type of feedstuff can affect this proportion (Sturkie, 1986). Farrell $(1974,1979)$, feeding a diet with 18.4 kJ gross energy (GE) observed that about $27 \%$ of the energy was lost in the feces and urine. Wiernusz (1994) also reported that, out of the total dietary ME content, only $38 \%$ is retained by the bird, whereas $34 \%$ is lost as heat and $28 \%$ as excreta (feces and urine) (Beker, 1979).

The evaluation of the energy content of poultry feeds is usually based on their content of metabolizable energy corrected for nitrogen (AMEn). However, the closest estimate of the "true" energy value of feed should be its NE content, which takes into account differences in the metabolic utilization of apparent metabolizable energy corrected for nitrogen (AMEn) and true metabolizable energy corrected for nitrogen (TMEn). Emmane (1994) proposed the effective energy system to define the energy content of feed ingredients and diets. The effective energy system takes into account differential heat increments, which are dependent on the catabolism of proteins vs. lipids in the 
body, and the variable efficiency of utilization and deposition of body lipids depends on whether (or not) they are derived from dietary lipids or synthesized from non-lipid material. He also suggested that the heat increment $(\mathrm{kJ} / \mathrm{d})$ of feeds is related to urinary nitrogen (6.98), fecal organ matter (0.91), positive nitrogen retention (8.72), positive lipid retention derived from dietary lipids (1.05), and positive lipid retention derived from non-lipid ingredients (3.92).

Metabolizable energy (ME) intake is partitioned into energy retained (ER) as fat and protein, and as heat production (HP: Heat Increments + Fasting Heat Production)(Close, 1990; Lawrence \& Fowler, 2002). Under thermoneutral conditions, HP represents the heat associated with the utilization of ME intake for maintenance (MEm) and productive processes, which in young broilers corresponds $52-64 \%$ of ME intake (Van Milgen et al., 2001; Noblet et al., 2003). Heat is produced by physical activity (AHP), thermal effect of feeding (TEF), and basal metabolic rate (fasting HP). Therefore, ER represents the difference between $M E$ and HP, or ER=ME-HP. (Van Milgen et al., 2001; Noblet et al., 2003).

Net energy is defined as ME minus the heat increment associated with the metabolic utilization of ME and the energy cost of ingestion, digestion, and some physical activity. It is calculated as the sum of (estimated or measured) fasting heat production and retained energy, where heat increment is the increase in heat production when food is ingested (Fraps, 1946). However, the $\mathrm{HI}$ of a given feed (as \% of ME) may not be constant over a broadrange of ME intake levels for a particular animal, and depends on several physiological factors. For instance, Heat Increment tends to be lower and not higher than maintenance energy supply (Noblet et al., 1993, 1994, 2007). The $\mathrm{HI}$ is also lower when ME is used for fat deposition compared with protein deposition (Noblet et al., 1999).

In a review article on poultry, Pirgozliev \& Rose (1999) compared the NE values of a series of feedstuffs as measured by Fraps (1946), with the values calculated according to different NE prediction equations. None of the NE systems for poultry found in literature was able to predict accurately the NE values measured in the feedstuffs; the widest discrepancy was observed with the system proposed by Emmans (1994). Choct et al. (2010) reported that energy loss as volatile fatty acids (VFA) was $101.5 \mathrm{~kJ} / \mathrm{chamber} / \mathrm{d}$ for birds fed a control diet compared with $34.3 \mathrm{~kJ} /$ chamber/d for those fed an enzyme-supplemented diet. Overall, xylanase reduced HP by $11 \%$ and decreased energy loss to excreta VFA by $66.2 \%$, showing that the enzyme promoted a higher NE (37\%) increase proportionally to AME (29.1\%). Carre et al. (2002) compared ME and NE levels for broilers and indicated that a large part of the difference between NE and ME was due to bird differences, and not to diet differences.

The present study aimed at determining the energy intake, utilization of AMEn and TMEn about NE, and dietaryenergy retention in commercial broilers.

\section{MATERIALS AND METHODS}

An experiment was conducted to evaluate the feed intake Fl; g), body weight gain (BWG; g), total energy intake $(\mathrm{kJ})$ and protein $(\mathrm{g})$ intake per metabolic body weight gain, energy efficiency ratio (EER), and protein efficiency ratio (PER), net energy and energy efficiency, and the effective energy in two commercial broiler strains (Arian and Ross 308).

Diets were formulated considering analyzed AMEn, TMEn, and nutrient levels of the feedstuffs (AOAC, 1990) in the laboratory of Animal Science Research Institute, I.R of Iran - Karaj, P. Box: 31585-1483 (Table 1). The treatments consisted of four diets formulated according to two different energy systems (AMEn and TMEn), and ontotal amino acid (TAA) or digestible amino acid(DAA) basis for the starter (1-21 days) and grower (22-42 days) phases (Table 2), as follows: AMEn $(\mathrm{kJ} / \mathrm{g})$ and total amino acid (TAA) levels (T1); AMEn $(\mathrm{kJ} / \mathrm{g})$ anddigestible amino acid levels (T2); TMEn $(\mathrm{kJ} / \mathrm{g})$ and total amino acid levels (T3); and TMEn ( $\mathrm{kJ} / \mathrm{g})$ and digestible amino acid (DAA) levels (T4), and two broiler strains (Arian and Ross 308).

In total, 960 broilers were distributed according to a completely randomized experimental design with a $2 \mathrm{x}$ 4 factorial arrangement ( 2 genetic strains and 4 diets) with four treatments and six replicates of 20 broilers each.

The experiment was carried out at the Animal Science Research Institute, Karaj, Iran. Broilers were housed in floor pens in an experimental broiler house with environmental control. Feed and water were offered ad libitum in tube feeders and nipple drinkers, respectively.

Feed intake and weight gain were recorded at the end of each phase. The protein efficiency ratio (PER) and the energy efficiency ratio (EER) were calculated for each phase. PER was calculatedas grams of weight gain /(CP\% intake * feed intake) * 100 . The EER was calculated in grams of (weight gain * 100)/total ME intake. ME intake was calculatedas a gram of weight gain / AMEn or TMEn intake * Feed Intake) * 100. 
NE was calculated using Eq. 1, 2 and 3, according to Geroote (1974a,b; 1975), MacLeod (2002) and Noblet et al., (1994, 2007, 2010, 2003) considering the chemical composition of the feedstuffs shown in Table 1.

$\mathrm{NE}(\mathrm{kJ} / \mathrm{g})=(\mathrm{AMEn}(0.60 * \mathrm{CP})+(0.90 * \mathrm{EE})+$ (0.75*NFE)/(CP+EE+NFE)

Eq 1 (Geroote, 1974a,b; 1975)

$\mathrm{NE}(\mathrm{kJ} / \mathrm{g})=\operatorname{AMEn}(0.60 * 17.8 \mathrm{CP})+(0.90 * 39.8 \mathrm{EE})+(0$. $75 * 17.7 \mathrm{NFE}) /(17.8 \mathrm{CP}+39.8 \mathrm{EE}+17.7 \mathrm{NFE})$

Eq. 2 (MacLeod, 2002)

$\mathrm{NE} \mathrm{kJ/g} \mathrm{=} \mathrm{AMEn} \mathrm{or} \mathrm{TMEn}-\mathrm{HI}$ Eq. 3

Heat Increment and efficiency of ME utilization for NE were adapted from Noblet et al., (1994, 2007, 2010, 2003).

Other nutrients included oyster shells, dicalcium phosphate, salt, vitamin and trace mineral premix, L-lysine, DL-methionine.

\section{Statistical analysis}

All data were analyzed for normal distribution using the normal option of the univariate procedure of GLM procedure of SAS software (SAS Inst. Inc., NC, USA). An entirely randomized design was employed. Data were analyzed using the GLM procedure. Duncan's multiple range test were used for comparison of the means $(p<0.05)$.

\section{RESULTS}

Feed intake, body weight, and the energy efficiency ratio (EER) were recordedat the end of each feeding phase (21 and 42 days) (Table 3 ). Results indicated that the main effect of dietary treatments on $\mathrm{Fl}, \mathrm{BW}$ and EER were significantly different $(p<0.05)$.

Feed intake and BW were considerably higher for dietary treatment 1 compared with other dietary treatments at 21 and 42 days of age $(p<0.05)$. AMEn and TMEn intake from 1 to 21 days did not affect EER results; however, from 22 and 42 days, there were significant differences among dietary treatments, with treatments 2 and 4 resulting in significantly higher EER than treatments 1 and $3(p<0.05)$.

The main differences between the two evaluated broiler strains were obtained for parameters $\mathrm{Fl}$, BW and EER $(p<0.05)$. Ross broilers presented significantly higher $\mathrm{Fl}, \mathrm{BW}$, and EER $(p<0.05)$ than the Arian strain.

Therefore, the results show that dietary AMEn and TMEn contents influenced both feed intake and body weight on days 21 and 42, but not energy efficiency ratio (EER). On the other hand, dietary amino acid expression significantly affected feed intake, body weight, and energy efficiency ratio (except for EER on day 21).

Table 1 - Nutritive values of the feedstuffs

\begin{tabular}{|c|c|c|c|c|c|c|c|c|c|c|}
\hline \multicolumn{3}{|c|}{$(\mathrm{kJ} / \mathrm{g})$} & \multicolumn{8}{|c|}{$\mathrm{g} / \mathrm{kg}$} \\
\hline & AMEn & TMEn & DM & $C P$ & $C F$ & $\mathrm{EE}$ & $\mathrm{Ca}$ & $P$ & Av. $P$ & Lino. Ac. \\
\hline Corn & 33.6 & 36.2 & 886.4 & 86.8 & 36 & 38 & 0.2 & 2.8 & 1 & 22 \\
\hline Soybean & 23.8 & 25.2 & 932.7 & 451 & 70 & 13 & 2.7 & 6.2 & 2.4 & 4 \\
\hline Soy oil & 88 & 91.9 & - & - & - & 1000 & - & - & - & 510 \\
\hline Fish meal & 21.9 & 29.8 & 93 & 52 & 7 & 94 & 22.9 & 17 & - & 15 \\
\hline
\end{tabular}

AMEn (apparent metabolizable energy corrected for nitrogen), TMEn (true metabolizable energy corrected for nitrogen), DM (dry matter), CP (crude protein), CF (crude fiber), EE (ether extract), Ca (calcium), P (phosphorus), Av.P (available phosphorus), Lino Ac.(Linoleic Acid)

Table 2 - Ingredients and calculated composition of the experimental diets according to feeding phase ( $\mathrm{g} / \mathrm{kg})$.

\begin{tabular}{|c|c|c|c|c|c|c|c|c|}
\hline \multirow[b]{2}{*}{ Treatments } & \multicolumn{4}{|c|}{ Diets 1 to 21 days } & \multicolumn{4}{|c|}{ Diets 22 to 42 days } \\
\hline & $\mathrm{T} 1$ & $\mathrm{~T} 2$ & T3 & T4 & $\mathrm{T} 1$ & $\mathrm{~T} 2$ & T3 & T4 \\
\hline Corn & 550 & 550 & 560 & 560 & 660 & 660 & 670 & 660 \\
\hline Soybean meal & 360 & 370 & 363.0 & 364.5 & 281.0 & 247.0 & 270 & 270 \\
\hline Fish meal & 34.0 & 21.0 & 30 & 25.4 & 10 & 40 & 20 & 18.5 \\
\hline Soybean oil & 31.7 & 30 & 6.5 & 7.0 & 32.0 & 34.0 & 7.0 & 10 \\
\hline Other Nutrients* & 24.3 & 29 & 40.5 & 43.1 & 17.0 & 19.0 & 33 & 41.5 \\
\hline \multicolumn{9}{|c|}{ Calculated nutrient (\%) and AMEn/TMEn content $(\mathrm{kJ} / \mathrm{g})$} \\
\hline AMEn or TMEn $(\mathrm{kJ} / \mathrm{g})$ & 12.80 & 15.48 & 12.93 & 12.97 & 13.35 & 13.47 & 13.51 & 13.47 \\
\hline $\mathrm{CP}$ & 229.0 & 228.9 & 229.9 & 230.4 & 189.7 & 189.8 & 190.8 & 190.6 \\
\hline DL-Met & 5.1 & 5.1 & 5.1 & 5.1 & 3.7 & 3.7 & 3.7 & 3.7 \\
\hline L-Lys & 14.4 & 14.4 & 14.4 & 14.4 & 10 & 10 & 10 & 10 \\
\hline
\end{tabular}

$\mathrm{T} 1=$ AMEn $(\mathrm{kJ} / \mathrm{g})+\mathrm{TAA}, \mathrm{T} 2=\mathrm{AMEn}(\mathrm{kJ} / \mathrm{g})+\mathrm{DAA}, \mathrm{T} 3=\mathrm{TMEn}(\mathrm{kJ} / \mathrm{g})+\mathrm{TAA}, \mathrm{T} 4=\mathrm{TMEn}(\mathrm{kJ} / \mathrm{g})+\mathrm{DAA}$. 
Table 3 - The effects of diet formulation based on AMEn or TMEn and TAA or DAA on the performance and energy efficiency ratio of Ross and Arian broilers.

\begin{tabular}{|c|c|c|c|c|c|c|}
\hline & \multicolumn{2}{|c|}{ EER\% } & \multicolumn{2}{|c|}{$B W G(g / d)$} & \multicolumn{2}{|c|}{$\mathrm{Fl}(\mathrm{g} / \mathrm{d})$} \\
\hline & 21D & $42 \mathrm{D}$ & 21D & $42 \mathrm{D}$ & $21 \mathrm{D}$ & $42 D$ \\
\hline \multicolumn{7}{|c|}{ Main effects of dietary treatment } \\
\hline 1 & $44.80 c \pm 3.34$ & $149.22 b \pm 12.31$ & $23.19 d \pm 0.84$ & $40.27 c \pm 1.42$ & $16.65 \pm 0.82$ & $8.33 c \pm 1.65$ \\
\hline 2 & $52.79 a \pm 4.38$ & $156.84 a \pm 11.50$ & $28.19 a \pm 2.30$ & $46.59 a \pm 3.51$ & $17.13 \pm 1.55$ & $9.19 a \pm 2.04$ \\
\hline 3 & $44.94 c \pm 1.32$ & $143.05 b \pm 13.15$ & $23.72 c \pm 0.54$ & $38.71 d \pm 0.99$ & $17.04 \pm 0.31$ & $8.46 b c \pm 0.59$ \\
\hline 4 & $50.57 \mathrm{~b} \pm 3.43$ & $147.86 b \pm 10.60$ & $26.48 b \pm 1.16$ & $42.23 b \pm 2.36$ & $16.98 \pm 0.9 .3$ & $8.90 a b \pm 0.99$ \\
\hline MSE & 12.460 & 155.49 & 1.49 & 6.14 & 1.08 & 0.36 \\
\hline \multicolumn{7}{|l|}{ Strains } \\
\hline Arian & $47.11 \mathrm{~b} \pm 5.65$ & $144.89 \mathrm{~b} \pm 18.13$ & $22.72 b \pm 5.38$ & $38.68 b \pm 8.15$ & $15.64 b \pm 2.26$ & $8.28 b \pm 0.92$ \\
\hline Ross & $49.67 a \pm 3.01$ & $154.47 a \pm 3.56$ & $28.60 a \pm 0.81$ & $45.87 a \pm 2.96$ & $18.54 a \pm 0.82$ & $9.24 a \pm 0.77$ \\
\hline \multicolumn{7}{|c|}{ Metabolizable energy } \\
\hline AMEn & $48.80 a \pm 5.36$ & $153.03 a \pm 18.13$ & $25.69 a \pm 5.38$ & $43.43 a \pm 8.15$ & $16.89 \pm 2.26$ & $8.76 \pm 0.92$ \\
\hline TMEn & $47.76 b \pm 2.37$ & $145.46 b \pm 13.28$ & $25.11 b \pm 1.31$ & $40.47 b \pm 2.1$ & $17.00 \pm 1.30$ & $8.68 \pm 0.64$ \\
\hline \multicolumn{7}{|c|}{ Amino acid expression } \\
\hline TAA & $44.87 \mathrm{~b} \pm 3.83$ & $146.14 b \pm 14.37$ & $23.46 b \pm 3.95$ & $39.49 b \pm 4.8$ & $16.84 \pm 2.38$ & $8.39 b \pm 0.61$ \\
\hline DAA & $51.68 a \pm 3.9$ & $152.35 \mathrm{a} \pm 14.37$ & $27.34 a \pm 3.95$ & $44.41 \mathrm{a} \pm 4.8$ & $18.05 \pm 2.38$ & $9.05 a \pm 0.32$ \\
\hline \multicolumn{7}{|c|}{ Interaction } \\
\hline$T * B$ & $<0.0001$ & $<0.0001$ & $<0.0001$ & $<0.0001$ & $<0.0001$ & 0.014 \\
\hline$T * E$ & $<0.0001$ & $<0.0001$ & $<0.0001$ & $<0.0001$ & $<0.0001$ & $<0.0001$ \\
\hline$T * A$ & $<0.0001$ & $<0.0001$ & $<0.0001$ & $<0.0001$ & $<0.0001$ & $<0.0001$ \\
\hline$B * E$ & $<0.0001$ & $<0.0001$ & $<0.0001$ & $<0.0001$ & $<0.0014$ & 0.006 \\
\hline$B * A$ & $<0.0001$ & 0.05 & $<0.0001$ & $<0.0001$ & $<0.0001$ & 0.51 \\
\hline$E * A$ & $<0.0001$ & 0.55 & $<0.0001$ & $<0.0001$ & 0.213 & 0.206 \\
\hline$T * E^{*} A$ & $<0.0001$ & $<0.0001$ & $<0.0001$ & $<0.0001$ & $<0.0001$ & $<0.0001$ \\
\hline$B * E * A$ & 0.08 & 0.73 & $<0.0001$ & $<0.0001$ & 0.013 & 0.08 \\
\hline
\end{tabular}

The effects of the treatments on protein efficiency ratio (PER), energy (AMEn/TMEn) intake, and protein intake on are shownin Table 4. PER was calculated as grams of weight gain per gram of protein intake. Dietary treatments significantly affected PER, as well as protein and energy intakes $(p<0.05)$ on day 42 , but not on day 21 . On day 42, PER was significantly higher $(p<0.05)$ in broilers fed dietary treatment 2 (AMEn and DAA), compared with dietary treatments 1 (AMEn and TAA) and 3 (TMEn and TAA).

The results of the experiment also show significant differences in PER, energy intake, and protein intake between genetic strains. Ross broilers showed higher PER at both ages, as well as lower energy and protein intakes $(p<0.05)$ than Arian birds. Interestingly, compared with Arian, Ross broilers presented higher EER and PER, but lower energy and protein intakes $(p<0.05)$.

The main effect of dietary AMEn and TMEn contents did not affect PER or energy and protein intakes from $d$ 21 to 42. The PER value significantly increased, whereas energy intake (AMEn and TMEn) and protein intake significantly decreased when broilers were fed diets formulated on digestible amino acid basis compared with total amino acid basis on day 42, but not on day 21 (Table 4).

Dietary treatments significantly $(p<0.05)$ influenced metabolic body weight gain and energy (AMEn or TMEn) intake as measured on d 21 and 42 (Table 5). The highest metabolic body weight gain $(p<0.05)$ was obtained with treatment 2 (AMEn and DAA).

Based on the metabolic body weight $\left(\mathrm{BW}^{0.75}\right)$ results, Ross broilers performed significantly better than Arian birds $(p<0.05)$. Energy intake in Arian broilers was significantly higher $(p<0.05)$ during both rearing phases compared with Ross broilers.

The results indicated that feeds formulated on AMEn or TMEn did not influence metabolic body weight on d 42. However, feeds formulated on DAA basis increased metabolic body weight and energy intake (AMEn and TMEn) on d21, but not on $\mathrm{d} 42 \mathrm{in}$ both broilers two strains $(p<0.05)$.

The main effect of dietary treatments significantly influenced EER/MEn intake per BWG and EER/ MEn intake per BWkg ${ }^{0.75}$ on days 21 and 42 (Table 6). There were no significant differences between treatments 3 and 4 relative to EER/AMEn intake per BWG. Treatment2 promoted significant higher EER/AMEn intake/BWG 
Table 4 - The effects of dietary AMEn and TMEn utilization in protein efficiency ratio, protein and ME intake in the diets of broilers (kJ/g)

\begin{tabular}{|c|c|c|c|c|c|c|}
\hline & \multicolumn{2}{|c|}{ Protein Intake } & \multicolumn{2}{|c|}{ AMEn or TMEn Intake/BWG } & \multicolumn{2}{|l|}{ PER\% } \\
\hline & $21 \mathrm{D}$ & $42 \mathrm{D}$ & 21D & $42 \mathrm{D}$ & $21 \mathrm{D}$ & $42 \mathrm{D}$ \\
\hline \multicolumn{7}{|c|}{ Main effects of dietary treatment } \\
\hline 1 & $2.24 \pm 0.11$ & $1.41 c \pm 0.12$ & $25.27 \pm 1.76$ & $49.24 a \pm 4.40$ & $0.45 \pm 0.03$ & $0.87 a \pm 0.07$ \\
\hline 2 & $2.31 \pm 0.20$ & $1.53 a \pm 0.06$ & $25.14 \pm 2.13$ & $144.06 b \pm 1.84$ & $0.44 \pm 0.04$ & $0.78 b \pm 0.03$ \\
\hline 3 & $2.29 \pm 0.04$ & $1.43 b c \pm 0.1$ & $24.60 \pm 0.46$ & $47.90 a \pm 3.34$ & $0.44 \pm 0.008$ & $0.85 a b \pm 0.06$ \\
\hline 4 & $2.28 \pm 0.12$ & $1.51 \mathrm{ab} \pm 0.07$ & $24.85 \pm 3.39$ & $45.39 b \pm 2.01$ & $0.43 \pm 0.02$ & $0.80 \mathrm{~b} \pm 0.04$ \\
\hline MSE & 0.52 & 0.01 & 0.15 & 0.64 & 0.0008 & 0.004 \\
\hline \multicolumn{7}{|l|}{ Strains } \\
\hline Arian & $2.11 b \pm 0.13$ & $1.40 b \pm 0.05$ & $29.90 a \pm 1.76$ & $48.91 \mathrm{a} \pm 1.80$ & $0.48 a \pm 0.03$ & $0.87 a \pm 0.03$ \\
\hline Ross & $2.50 \mathrm{a} \pm 0.05$ & $1.57 a \pm 0.13$ & $22.68 \mathrm{~b} \pm 0.88$ & $43.93 \mathrm{~b} \pm 3.93$ & $0.40 b \pm 0.02$ & $0.78 b \pm 0.07$ \\
\hline \multicolumn{7}{|c|}{ Metabolizable energy } \\
\hline AMEn & $2.27 \pm 0.3$ & $1.48 \pm 0.15$ & $25.23 \pm 3.39$ & $46.61 \pm 4.98$ & $0.45 \pm 0.06$ & $0.82 \pm 0.09$ \\
\hline TMEn & $2.29 \pm 0.17$ & $1.47 \pm 0.10$ & $24.73 \pm 1.90$ & $46.65 \pm 3.35$ & $0.44 \pm 0.03$ & $0.83 \pm 0.06$ \\
\hline \multicolumn{7}{|c|}{ Amino acid } \\
\hline TAA & $2.27 \pm 0.15$ & $1.42 b \pm 0.16$ & $24.94 \pm 1.76$ & $48.57 a \pm 5.56$ & $0.44 \pm 0.03$ & $0.86 a \pm 0.09$ \\
\hline DAA & $2.29 \pm 0.32$ & $1.53 a \pm 0.10$ & $24.98 \pm 0.75$ & $44.68 b \pm 2.88$ & $0.44 \pm 0.06$ & $0.79 b \pm 0.05$ \\
\hline \multicolumn{7}{|c|}{ Interaction } \\
\hline$T * B$ & $<0.0001$ & 0.015 & $<0.0001$ & 0.003 & 0.0002 & 0.003 \\
\hline$T * E$ & $<0.0001$ & $<0.0001$ & $<0.0001$ & $<0.0001$ & $<0.0001$ & $<0.0001$ \\
\hline$T * A$ & $<0.0001$ & $<0.0001$ & $<0.0001$ & $<0.0001$ & $<0.0001$ & $<0.0001$ \\
\hline$B * E$ & 0.0013 & 0.007 & 0.004 & 0.002 & 0.007 & 0.002 \\
\hline$B * A$ & $<0.0001$ & 0.51 & 0.0002 & 0.30 & 0.0006 & 0.32 \\
\hline$E^{\star} A$ & 0.21 & 0.21 & 0.58 & 0.12 & 0.55 & 0.12 \\
\hline$T * E * A$ & $<0.0001$ & $<0.0001$ & $<0.0001$ & $<0.0001$ & $<0.0001$ & $<0.0001$ \\
\hline$B * E^{*} A$ & 0.015 & 0.07 & 0.06 & 0.03 & 0.07 & 0.03 \\
\hline
\end{tabular}

Table 5 - The effects of dietary AMEn and TMEn utilization in BW ${ }^{0.75}$ ME intake in broiler diets $(\mathrm{kJ} / \mathrm{g})$

\begin{tabular}{|c|c|c|c|c|}
\hline & \multicolumn{2}{|c|}{ AMEn or TMEn Intake/BW0.75 } & \multicolumn{2}{|c|}{ Metabolic body weight (BW 0.75) } \\
\hline & $21 \mathrm{D}$ & $42 \mathrm{D}$ & $21 \mathrm{D}$ & $42 \mathrm{D}$ \\
\hline \multicolumn{5}{|c|}{ Main effects of dietary treatments } \\
\hline 1 & $10.54 d \pm 0.3$ & $15.91 c \pm 0.42$ & $55.18 a b \pm 3.05$ & $122.76 a \pm 10.30$ \\
\hline 2 & $12.18 \mathrm{a} \pm 0.73$ & $17.79 a \pm 0.005$ & $57.19 a \pm 4.22$ & $114.56 b \pm 4.60$ \\
\hline 3 & $10.75 c \pm 0.18$ & $15.51 d \pm 0.59$ & $54.27 b \pm 1.05$ & $119.49 a b \pm 0.25$ \\
\hline 4 & $11.67 \mathrm{~b} \pm 0.39$ & $16.56 \mathrm{~b} \pm 0.7$ & $56.31 \mathrm{ab} \pm 2.97$ & $115.73 a b \pm 5.44$ \\
\hline MSE & 0.21 & 0.52 & 0.66 & 3.95 \\
\hline \multicolumn{5}{|l|}{ Birds } \\
\hline Arian & $10.40 \mathrm{~b} \pm 0.39$ & $15.49 \mathrm{~b} \pm 0.51$ & $58.66 a \pm 3.8$ & $121.58 a \pm 4.64$ \\
\hline Ross & $12.35 a \pm 0.41$ & $17.60 \mathrm{a} \pm 0.85$ & $52.3 b \pm 1.9$ & $113.97 b \pm 9.83$ \\
\hline \multicolumn{5}{|c|}{ Metabolizable energy } \\
\hline AMEn & $11.36 a \pm 1.78$ & $16.68 a \pm 2.38$ & $56.20 \pm 5.65$ & $119.79 \pm 8.66$ \\
\hline TMEn & $11.21 \mathrm{~b} \pm 0.44$ & $15.72 b \pm 0.62$ & $55.31 \pm 3.64$ & $117.61 \pm 8.70$ \\
\hline \multicolumn{5}{|c|}{ Amino acid } \\
\hline TAA & $10.64 b \pm 0.95$ & $15.72 \mathrm{~b} \pm 1.63$ & $54.77 \mathrm{~b} \pm 3.09$ & $121.13 a \pm 11.13$ \\
\hline DAA & $11.93 a \pm 1.29$ & $16.57 a \pm 1.38$ & $56.77 a \pm 6.19$ & $115.14 b \pm 6.27$ \\
\hline \multicolumn{5}{|c|}{ Interaction } \\
\hline $\mathrm{T} * \mathrm{~B}$ & $<0.0001$ & $<0.0001$ & 0.002 & 0.44 \\
\hline$T \star E$ & $<0.0001$ & $<0.0001$ & $<0.0001$ & $<0.0001$ \\
\hline$T^{*} A$ & $<0.0001$ & $<0.0001$ & $<0.0001$ & $<0.0001$ \\
\hline$B * E$ & $<0.0001$ & $<0.0001$ & 0.58 & 0.44 \\
\hline$B * A$ & $<0.0001$ & $<0.0001$ & 0.0005 & 0.85 \\
\hline$E^{*} A$ & $<0.0001$ & $<0.0001$ & 0.97 & 0.38 \\
\hline$T * E * A$ & $<0.0001$ & $<0.0001$ & $<0.0001$ & $<0.0001$ \\
\hline$B * E * A$ & $<0.0001$ & $<0.0001$ & 0.11 & 0.15 \\
\hline
\end{tabular}


Table 6 - The effects of dietary AMEn and TMEn utilization in metabolic body weight (BW 0.75 , AMEn and TMEn intake/ $\mathrm{BW}^{0.75}$, EER/MEni and TMEni /BWG, EER/ BW ${ }^{0.75}$ in broiler diets

\begin{tabular}{|c|c|c|c|c|}
\hline & \multicolumn{2}{|c|}{ EER/MEn intake/BW0.75 } & \multicolumn{2}{|c|}{ EER/MEn intake/BWG } \\
\hline & 21 & 42 & 21 & 42 \\
\hline \multicolumn{5}{|c|}{ Main effects of treatments } \\
\hline 1 & $2.79 b \pm 0.26$ & $0.73 c \pm 0.07$ & $1.27 \pm 0.02$ & $0.29 b \pm 0.05$ \\
\hline 2 & $3.02 \mathrm{a} \pm 0.56$ & $0.88 a \pm 0.07$ & $1.30 \pm 0.2$ & $0.34 \mathrm{a} \pm 0.04$ \\
\hline 3 & $2.91 \mathrm{ab} \pm 0.11$ & $0.75 b c \pm 0.1$ & $1.32 \pm 0.05$ & $0.30 a b \pm 0.02$ \\
\hline 4 & $2.90 \mathrm{ab} \pm 0.35$ & $0.82 a b \pm 0.08$ & $1.28 \pm 0.13$ & $0.32 a b \pm 0.03$ \\
\hline MSE & 0.13 & 0.01 & 0.02 & 0.002 \\
\hline \multicolumn{5}{|l|}{ Strain } \\
\hline Arian & $2.46 b \pm 0.30$ & $0.71 b \pm 0.05$ & $1.13 b \pm 0.14$ & $0.29 b \pm 0.02$ \\
\hline Ross 308 & $3.45 a \pm 0.32$ & $0.89 a \pm 0.14$ & $1.49 a \pm 0.12$ & $0.34 a \pm 0.05$ \\
\hline \multicolumn{5}{|c|}{ Metabolizable energy } \\
\hline AMEn & $2.90 \pm 0.77$ & $0.81 \pm 0.16$ & $1.28 \pm 0.29$ & $0.31 \pm 0.05$ \\
\hline TMEn & $2.91 \pm 0.43$ & $0.78 \pm 0.12$ & $1.30 \pm 0.18$ & $0.31 \pm 0.04$ \\
\hline \multicolumn{5}{|c|}{ Amino acid } \\
\hline TAA & $2.85 \pm 0.38$ & $0.74 b \pm 0.17$ & $1.29 \pm 0.15$ & $0.29 b \pm 0.06$ \\
\hline DAA & $2.96 \pm 0.83$ & $0.81 a \pm 0.12$ & $1.28 \pm 0.32$ & $0.33 a \pm 0.04$ \\
\hline$T * B$ & $<0.0001$ & 0.025 & 0.0001 & 0.26 \\
\hline \multicolumn{5}{|c|}{ Interaction } \\
\hline$T \star E$ & $<0.0001$ & $<0.0001$ & $<0.0001$ & $<0.0001$ \\
\hline$T * A$ & $<0.0001$ & $<0.0001$ & $<0.0001$ & $<0.0001$ \\
\hline$B \star E$ & 0.0013 & 0.008 & 0.094 & 0.14 \\
\hline$B^{*} A$ & $<0.0001$ & 0.62 & $<0.0001$ & 0.83 \\
\hline$E^{*} A$ & 0.12 & 0.22 & 0.38 & 0.37 \\
\hline$T * E * A$ & $<0.0001$ & $<0.0001$ & $<0.0001$ & $<0.0001$ \\
\hline$B * E * A$ & 0.008 & 0.13 & 0.03 & 0.17 \\
\hline
\end{tabular}

than treatment 2 on $d 21$ and $42(p<0.05)$. On the other hand, treatments had no effect on EER/ AMEn / BW 0.75 on day 21. On day 42, EER/AMEn / BW 0.75 was not different between treatments 3 and 4 , but higher in treatment 2 compared with treatment $1(p<0.05)$.

Ross broilers presented higher EER/MEn intake per BWG and EER/ MEn intake per BWkg ${ }^{0.75}$ on days 21 and 42 compared with Arian $(p<0.05)$.

The results show that energy values had no effect on the EER/MEn intake per BWG and EER/ MEn intake per $\mathrm{BWkg}^{0.75}$ on $\mathrm{d} 21$ or 42 . Also, the amino acid concept had no effect on EER/MEn intake per BWG and EER/ MEn intake per BWkg ${ }^{0.75}$ on d 21, where feeds formulated on DAA promoted higher EER/MEn intake per BWG and EER/ MEn intake per BWkg ${ }^{0.75}$ on d 42 ( $p<0.05)$.

Table 7 shows daily NE, NE per AMEn or TMEn intake, and $\mathrm{HI}$ results obtained on days 21 and 42 based on metabolic body weight $\left(\mathrm{kg}^{0.75}\right)$ to eliminate the effect of body weight change per $\mathrm{kg}^{0.75}$ birds resulting from dietary AMEn and TMEn. Dietary treatments had no impact on NE on d 21, but on d 42, treatment 2 promoted significantly higher NE than treatment 4 . There were no significant effects of dietary treatment on NE/AMEn and TMEn intake on d 21 and 42, but affected heat increment efficiency (HI/AMEn) on d 42, but not on d 21.

Genetic strain affected NE and NE/AMEn or TMEn results $(p<0.05)$. Ross broiler presented lower NE values on $d 21$ and 42, lower NE/AMEn or TMEn on d 21, and higher HI/AMEn on $\mathrm{d} 21$ and 42.

Dietary AMEn and TMEn values did not significantly affect NE on d 21,NE/AMEn or TMEn on d 21 or 42 days, or HI/AMEn on d 21. However, dietary TMEn reduced NE and increased HI/AMEn intake on d 42. On the other hand, amino acid formulation did not affect NE, NE/AMEn or TMEn, or and HI/AMEn, except for HI/ AMEn on $d 42$, when broilers fed diets based on TAA presented higher HI/AMEn intake about DAA.

The calculated NE values of the treatment diet for 21 - and 42-d-old broilers are shown in Table 8. The NE3 values, calculated using the equation based on Noblet, were higher than theNE1, and NE2 values obtained according to the equations of MacLeod (2002) and Geroote (1975).

The differences and ratios between the NE values calculated by the different equations are presented in Table 9 and 10. The differences between calculated NE 
Table 7 - Efficiency of dietary AMEn and TMEn utilization for net energy (NE) in broiler

\begin{tabular}{|c|c|c|c|c|c|c|}
\hline & \multicolumn{2}{|l|}{ HI/AMEn } & \multicolumn{2}{|c|}{ NE/AMEn or TMEn } & \multicolumn{2}{|l|}{$\mathrm{NE}(\mathrm{kJ} / \mathrm{g})$} \\
\hline & $21 \mathrm{D}$ & $42 \mathrm{D}$ & 21D & $42 \mathrm{D}$ & $21 \mathrm{D}$ & $42 \mathrm{D}$ \\
\hline \multicolumn{7}{|c|}{ Main effects of dietary treatment } \\
\hline 1 & $74.85 \pm 9.54$ & $125.81 \mathrm{ab} \pm 16.27$ & $90.64 \pm 0.07$ & $92.76 \pm 8.76$ & $0.56 \pm 0.07$ & $0.32 c \pm 0.03$ \\
\hline 2 & $79.25 \pm 6.07$ & $131.17 a \pm 14.10$ & $89.99 \pm 0.04$ & $93.48 \pm 0.02$ & $0.54 \pm 0.04$ & $0.32 c \pm 0.03$ \\
\hline 3 & $73.13 \pm 1.84$ & $120.50 b c \pm 7.53$ & $85.76 \pm 0.01$ & $91.59 \pm 0.03$ & $0.57 \pm 0.01$ & $0.35 b \pm 0.03$ \\
\hline 4 & $76.86 \pm 3.97$ & $114.97 c \pm 6.02$ & $85.39 \pm 0.02$ & $90.88 \pm 0.02$ & $0.55 \pm 0.02$ & $0.37 a \pm 0.02$ \\
\hline MSE & 3.12 & 5.15 & 0.003 & 3.25 & 0.003 & 0.00007 \\
\hline \multicolumn{7}{|l|}{ Strains } \\
\hline Arian & $81.92 a \pm 10.12$ & $133.35 \mathrm{a} \pm 5.90$ & $99.48 \mathrm{a} \pm 0.07$ & $99.69 \pm 0.01$ & $0.51 b \pm 0.06$ & $0.31 b \pm 0.01$ \\
\hline Ross & $68.91 b \pm 2.38$ & $110.83 b \pm 18.20$ & $99.39 \mathrm{~b} \pm 0.02$ & $97.67 \pm 6.2$ & $0.60 a \pm 0.02$ & $0.37 a \pm 0.04$ \\
\hline \multicolumn{7}{|c|}{ Metabolisable energy } \\
\hline AMEn & $77.03 \pm 13.85$ & $128.49 \mathrm{a} \pm 18.24$ & $99.44 \pm 0.09$ & $97.90 \pm 5.91$ & $0.56 \pm 0.09$ & $0.32 b \pm 0.03$ \\
\hline TMEn & $74.98 \pm 7.11$ & $117.74 b \pm 19.99$ & $99.44 \pm 0.05$ & $99.63 \pm 0.06$ & $0.55 \pm 0.05$ & $0.36 a \pm 0.06$ \\
\hline \multicolumn{7}{|c|}{ Amino acid } \\
\hline TAA & $73.97 \pm 8.87$ & $123.13 \pm 19.87$ & $99.43 \pm 0.07$ & $97.88 \pm 5.92$ & $0.57 \pm 0.07$ & $0.34 b \pm 0.05$ \\
\hline DAA & $78.07 \pm 11.29$ & $123.09 \pm 17.70$ & $99.45 \pm 0.075$ & $99.65 \pm 0.05$ & $0.54 \pm 0.07$ & $0.35 a \pm 0.05$ \\
\hline \multicolumn{7}{|c|}{ Interaction } \\
\hline$T * B$ & 0.07 & $<0.0001$ & 0.04 & 0.31 & 0.04 & $<0.0001$ \\
\hline$T * E$ & $<0.0001$ & $<0.0001$ & $<0.0001$ & $<0.0001$ & $<0.0001$ & $<0.0001$ \\
\hline$T * A$ & $<0.0001$ & $<0.0001$ & $<0.0001$ & $<0.0001$ & $<0.0001$ & $<0.0001$ \\
\hline$B * E$ & 0.64 & $<0.0001$ & 0.90 & 0.28 & 0.9 & $<0.0001$ \\
\hline$B^{*} A$ & 0.02 & 0.035 & 0.01 & 0.26 & 0.01 & 0.07 \\
\hline$E^{*} A$ & 0.88 & 0.065 & 0.84 & 0.31 & 0.84 & 0.0003 \\
\hline$T * E * A$ & $<0.0001$ & $<0.0001$ & $<0.0001$ & $<0.0001$ & $<0.0001$ & $<0.0001$ \\
\hline$B * E * A$ & 0.25 & 0.005 & 0.15 & 0.26 & 0.15 & $<0.0001$ \\
\hline
\end{tabular}

values obtained by the various equations are not similar to the differences (Table 9 and 10). Data show that on $d$ 21 and 42, the differences between NE values obtained from Eq.1, and 2 were not similar to differences in Eq.3 (NE calculated) to1 and2 (see Tables 9 and 10). As well as, different between NE values obtained from equation 3 (NE calculated), and 2 (MacLeod 2002)were higher than those between equation 1 and 2 .

The NE/AMEn and TMEn efficiency ratios obtained using NE3 (calculated as MEn or TMEn - HI) were higher compared with those achieved with NE1 (Geroote 1975) and NE2 (MacLeod 2002). The numerical difference in NE/AMEn and TMEn efficiency between equation 1 and 2 was about 8 - $18 \%$ for AMEn and approximately $14 \%$ for TMEn (Table 9 ). The NE/AMEn and TMEn efficiency difference between equation 3 and 1 was estimatedatabout 18\% for AMEn and 12\% for TMEn on d 42.

Interestingly, the NE/AMEn and TMEn efficiency calculated by equations 1 and 2 were higher in T1 (AMEn+TAA) and T2 (AMEn+DAA) than in T3 (TMEn+TAA) and T4 (TMEn+DAA). On the other hand, according to Table 10, NE/AMEn and TMEn efficiency values calculated by equation 3 .

Effective energy as a system is analogous to productive or net energy system in that it attempts

Table 8 - Mean and SD NE values of dietary energy (AMEn and TMEn) in 21- and 42-d-old broilers.

\begin{tabular}{lllllllll}
\hline & & \multicolumn{9}{c}{$42 \mathrm{D}(\mathrm{kJ} / \mathrm{g})$} & & NE3 & S $(\mathrm{kJ} / \mathrm{g})$ \\
\hline T1 & NE1 & NE2 & NE3 & SD & NE1 & NE2 & NE3 \\
T2 & 11.38 & 9.16 & 12.34 & 0.40 & 12.17 & 9.66 & 12.88 & 0.40 \\
T3 & 11.29 & 9.20 & 12.38 & 0.39 & 12.34 & 9.79 & 13.01 & 0.41 \\
T4 & 11.04 & 9.79 & 13.26 & 0.39 & 11.63 & 10.25 & 13.81 & 0.40 \\
\hline
\end{tabular}

T1=AMEn+TAA, T2=AMEn+DAA, T3=TMEn+TAA, T4=TMEn+DAA

$\mathrm{NE} 1=$ Geroote $\left.1975(\mathrm{~kJ} / \mathrm{g}) \mathrm{NE}=\left(\mathrm{AMEnorTMEn}{ }^{*}\left(0.6^{*} \mathrm{CP}\right)+\left(0.9^{*} \mathrm{EE}\right)+\left(0.75^{*} \mathrm{NFE}\right)\right) /(\mathrm{CP}+\mathrm{EE}+\mathrm{NFE})\right)$

NE2=MacLeod $2002(\mathrm{~kJ} / \mathrm{g})$

(AMEn orTMEn $\left.\left(0.6^{*}\left(4.26^{*} \mathrm{CP}\right)+\left(0.9^{*}\left(9.5^{*} \mathrm{EE}\right)+\left(0.75^{*}\left(4.23^{*} \mathrm{NFE}\right)\right)\right)\right) /\left(4.26^{*} \mathrm{CP}\right)+\left(9.5^{*} \mathrm{EE}\right)+\left(4.23^{*} \mathrm{NFE}\right)\right) / 100$

NE3= calculated as MEn or TMEn $-H$, adapted from Noblet et al., $(1994,2007,2010,2003)$. 
Table 9 - Efficiency of AMEn and TMEn transformation into NE in the experimental diets, as calculated by equations 1 and 2.

\begin{tabular}{llllllll}
\hline & \multicolumn{3}{c}{$42 \mathrm{D}(\mathrm{kJ} / \mathrm{g})$} & & $21 \mathrm{D}(\mathrm{kJ} / \mathrm{g})$ \\
\hline T1 & NE1-NE2 & NE1/ME & NE2/ME & NE1-NE2 & NE1/ME & NE2/ME & SD \\
T2 & 77.21 & 88.96 & 71.69 & 86.36 & 91.29 & 72.45 & 32.57 \\
T3 & 49.04 & 88.06 & 71.64 & 86.69 & 92.36 & 73.22 & 32.84 \\
T4 & 46.40 & 80.72 & 71.26 & 49.49 & 81.63 & 71.97 & 30.46 \\
\hline
\end{tabular}

T1=AMEn+TAA, T2=AMEn+DAA, T3=TMEn+DAA, T4=TMEn+DAA

Table 10 - Efficiency of AMEn and TMEn transformation into NE in the experimental diets, as calculated by equation 3.

\begin{tabular}{llllllll}
\hline & 42 D $(\mathrm{kJ} / \mathrm{g})$ & 21 D $(\mathrm{kJ} / \mathrm{g})$ & \multicolumn{3}{c}{ 42 D $(\mathrm{kJ} / \mathrm{g})$} & $\mathrm{N}(\mathrm{kJ} / \mathrm{g})$ & \\
\hline & NE3-NE1 & NE3-NE2 & NE3-NE1 & NE3-NE2 & NE3/ME & NE3/ME & SD \\
\hline T1 & 32.63 & 107.46 & 23.09 & 104.68 & 96.49 & 96.63 & 42.47 \\
T2 & 36.57 & 107.78 & 22.34 & 104.39 & 96.50 & 97.57 & 42.58 \\
T3 & 69.24 & 110.16 & 65.73 & 107.44 & 96.72 & 96.85 & 38.12 \\
T4 & 71.84 & 110.25 & 64.73 & 107.23 & 96.74 & 96.86 & 37.97 \\
\hline
\end{tabular}

to categorize heat increment. As data in Table 11 indicates, the effective energy (EE) value of dietary treatments observed on d 21 (90\%) was numerically lower than that obtained on d 42 (93\%). However, effective energy (EE) value obtained from diets include with AMEn and TMEn (T1 and T4) were not numerically different changeable for two strains of broiler from 21 and 42 days (Table 11).

\section{DISCUSSION}

Our findings showed that the diet formulated on apparent metabolizable energy and digestible amino acid level (T2) promoted significantly better Fl, body weight, PER, and EER results in 42-d-old broilers. Compared with Arian broilers, Ross birds displayed a significantly higher FI and BW. The formulation of diets based on AMEn or TMEn did not influence EER, PER, or the ratio of $\mathrm{ME}$ intake per BWG and $\mathrm{BW}^{0.75}$.

Protein and energy efficiencies were reduced between the starter (21 days) and the finisher (42 days) periods. Energy is not used $100 \%$ efficiently for production in poultry because, during metabolism, around $15 \%$ of the energy is wasted as heat increment or specific dynamic action. The most significant sources of heat increment in the gut are associated with digestion, secretion, absorption, and metabolism of the digest. This is shown by the HI/ME (AMEn and TMEn) intake results. This ratio was significantly higher in treatment 4 (DAA + TMEn) compared with the other treatments on $\mathrm{d} 42$. The $\mathrm{HI}$ of a given feed (as \% of $\mathrm{ME}$ ) may not be constant over a broad range of $\mathrm{ME}$ intakes for a particular bird and depends on several physiological factors (Noblet et al., 1993; 1994; 2003; 2007; 2010).

The calculated effective energy for AMEn and TMEn was higher on $\mathrm{d} 42$ than on $\mathrm{d} 21$, and than the diet based on TMEn increased the effective energy of diet. No studies in the literature on NE systems for poultry were able to predict the analyzed NE values for feedstuffs accurately; the highest discrepancy was observed with the system proposed by Emmans (1994).

However, the formulation of feeds based on AMEn or TMEn did not significantly affect the NE / AMEn and TMEn ratio. It was reported that about $84 \%$ of the $\mathrm{ME}$ is available as NE, although birds factors and/or type of feedstuff may impact this proportion (Sturkie, 1986, Farrell, 1974). The efficiency of utilization of AME and TME for NE was calculated from the NE equation of Noblet et al. (1994, 2003, 2010). According to the NE/

Table 11 - Calculated effective energy (EE)* expressed as \% or (kJ/g) of AMEn and TMEn.

\begin{tabular}{|c|c|c|c|c|c|c|}
\hline & \multicolumn{4}{|c|}{42 days } & \multicolumn{2}{|l|}{21 days } \\
\hline & Arian & Ross & SD & Arian & Ross & SD \\
\hline T1 & $90.43(11.59)$ & $90.55(11.63)$ & 0.08 & $92.75(12.38)$ & $92.75(12.38)$ & 0.004 \\
\hline $\mathrm{T} 2$ & $90.52(11.63)$ & $90.34(11.50)$ & 0.13 & $92.97(12.51)$ & $92.82(12.42)$ & 0.11 \\
\hline T3 & $90.66(11.71)$ & $90.82(11.80)$ & 0.12 & $93.01(12.55)$ & $93.01(12.55)$ & 0.002 \\
\hline T4 & $90.73(11.76)$ & $90.79(11.80)$ & 0.04 & $92.95(12.51)$ & $93.04(12.55)$ & 0.06 \\
\hline
\end{tabular}

* Emmans (1994) described the derivation of a simple equation for estimation of effective energy by the following equation: Effective energy ( $\mathrm{kJ} / \mathrm{g})=\left(\left(\left(1.17{ }^{*} \mathrm{AMEn}\right.\right.\right.$ or TMEn)$\left.-\left(4.2^{*}(\mathrm{P})-2.44\right)\right) /\left(\mathrm{AMEn}\right.$ or TMEn) ${ }^{*} 100$ 
ME efficiency provided by Carre et al., (2002) for the main nutrients, the impact of ME on NE for estimating the energy value of ingredients. Also Carre et al., (2002) as compared ME to NE and showed a significant part of the differentiation between NE, and ME came from birds, not from diets.

The obtained nutritional NE values calculated from AMEn and TMEn and the efficiency of transformation AMEn and TMEn into NE calculated showed variations among dietary treatments. These results are consistent with Wiernusz (1994), who reported that, out of total feed energy, only $38 \%$ is retained by broilers, whereas $34 \%$ is lostto heat and another $28 \%$ is lost as excreta (feces and urine). Pirgozliev \& Rose (1999) compared the NE value of a series of feedstuffs, as measured by Fraps (1946).

In conclusion, the present study indicated that the formulation of diets on DAA and TMEn improved the utilization of net energy in 42-d-oldbroilers. Diet formulation with oil inclusion had positive effects on dietary NE level and the efficiency of dietary AMEn and TMEn for net energy utilization (NE). Future studies are needed to provide a better understanding of the interactions among nutrients and their effects within the lumen of the digestive tract, to propose more precise NE prediction equations for poultry.

This experiment indicated that NE is a better predictor of the energy value of broiler feeds than AMEn or TMEn because the results showed that AMEn significantly increased dietary NE and $\mathrm{HI}$ values and improved the efficiency of energy utilization.

Each equation used to calculate NE includes a broad range of bird and feed composition factors, which significantly influences the determination of $\mathrm{NE}$, efficiency of ME transformation in $\mathrm{NE}$, and $\mathrm{HI}$ values. In fact, the main result of this experiment is that bird variability accounted for a large part of the difference between NE and ME, and not diet formulation. Thus, the main point that remains to be predicted is the bird variability.

Finally, even though NE is the final objective of the energy evaluation of feeds, more attention should be lent to the accurate estimation of AMEn or TMEn values, which are the most important factors of variation of the energy value of poultry feeds. However, we used different equations to calculate net energy (NE) values. The data showed that the NE values of two commercial broiler diets are different. In contrast with Noblet et al. (1994), who reported that the energy cost of growth is independent of diet composition when expressed on NE basis, the results of the present experiment show that dietary NE values are related to diet composition. Thus, we suggest that the NE energy system should be used in broiler diet formulation.

\section{REFERENCES}

AOAC- Association of Official Analytical Chemists. Official method of analysis. 15 th ed. Arlington; 1990.

Becker WA, Spencer JV, Mirosh LW, Verstrate JA. Prediction of fat and fatfree live weight in broiler chickens using backskin fat, abdominal fat and live body weight. Poultry Science 1979;58:835-842.

Carre $B$, Lessir $M$, Juin $H$. Development of the energy system for broiler. Proceeding of the 38th Eastern Nutrition Conference; 2002; Guelph, Canada: ANAC; 2002. p. 140-149.

Choct M, Tukei A, Cadogan DJ. Non-starch polysaccharides and enzyme application influence the net energy value of broiler diets. Proceedings of the 21th Australian Poultry Science Symposium; 2010 Feb 1-3; Sidney, New South Wales; 2010. p.50.

Close WH. The evaluation of feeds through calorimetry studies. In: Wiseman J, Cole DJA, editors. Feedstuff evaluation. Cambridge: Butterworths; 1990. p.21-39

Emmans GC. Effective energy: a concept of energy utilization applied across species. British Journal of Nutrition 1994;171:801-821.

Farrell DJ. Energy systems for pigs and poultry: a review. Journal of the Australian Institute of Agricultural Science 1979;21-34.

Farrell DJ. Principles and assumptions of calorimetry. In: Morris TR, Freeman BM, editors. Energy requirements of poultry. Edinburgh: British Poultry Science; 1974. p.1-24.

Fraps GS. Composition and productive energy of poultry feeds and rations [bulletin 678]. Texas: Texas Agricultural Experiment Station; 1946.

Groote G de. Utilization of metabolisable energy. In: In: Morris TR, Freeman BM, editors. Energy requirements of poultry. Edinburgh: British Poultry Science; 1974a. p.113-134.

Groote $G$ de. A comparison of a new Net Energy system with the metabolisable energy system in broiler diet formulation, performance and profitability. British Poultry Science 1974b;15:75-95.

Groote G de. Net Energy systems for chickens. Proceedings of the Georgia Nutrition Conference; 1975; Atlanta, Georgia: Universty of Georgia; 1975. p.9-30

Lawrence TLL, Fowler VR. Efficiency and growth. In: Lawrence TL, Fowler VR, editors. Growth of farm animals. Wallingford: $C A B$ International; 2002. p.216-228.

Macleod MG. Energy utilization: measurement and prediction. In: McNab JM, Boorman KN, editors. Poultry feedstuffs: supply, composition and nutritive value. Edinburgh: Roslin Institute; 2002. p.181.

Noblet J, Dubois S, van Milgen J, Warpechowski M, Carre B. Heat production in broilers is not affected by dietary crude protein. In: Ortigues-Marty I, Miraux N, Brand-Williams S, editors. Energy and production metabolism and nutrition. Wageningen: Wageningen Academic Publishers; 2007. p.479-480.

Noblet J, Fortune H, Shi XS, Dubois S. Prediction of net energy value of feeds for growing pigs. Journal Animal Science 1994;72:344-354.

Noblet J, Henry Y. Energy evaluation systems for pig diets: A review. Livestock Production Science 1993;36:121- 141. 


\section{Broiler Diets}

Noblet J, Van Milgen J, Carre B, Dimon PS, Rademacher M, Van Cauwenderghe $\mathrm{S}$. Progress in research on energy and protein metabolism [EAAP publication 109]. Wageningen: Wageningen Academic Publishers; 2003. p.205-208.

Noblet J, Van Milgen J, Dubois S. Utilization of metabolisable energy of feed in pig and poultry: Interest of net energy systems? Australian Poultry Science Symposium 2010;21: 26-43.

Pirgozliev V, Ross SP. Net energy system for poultry feeds: a quantitative review. Worlds Poultry Science Journal 1999;55:23-36.
Sturkie PD. Avian physiology. New York: Springer-Verlag; 1986.

Van Milgen J, Noblet J, Dubois S, Carre B, Juin H. Utilization of metabolisable energy in broiler chickens. Poultry Science 2001;80(Suppl.1):170.

Wiernusz CJ. Energetic efficiency of substrate source as related to broiler protein and lipid accretion [dissertation]. Stillwater: Oklahoma State University; 1994. 